Article

\title{
Determining the Frequency for Load-Independent Output Current in Three-Coil Wireless Power Transfer System
}

\author{
Longzhao Sun *, Houjun Tang and Yingyi Zhang \\ Department of Electrical Engineering, Shanghai Jiao Tong University, Shanghai 200240, China; \\ E-Mails: hjtang@sjtu.edu.cn (H.T.); Rache10810@sjtu.edu.cn (Y.Z.) \\ * Author to whom correspondence should be addressed; E-Mail: sunlongzhao@sjtu.edu.cn; \\ Tel.: +86-150-0086-2302.
}

Academic Editor: K. T. Chau

Received: 29 July 2015 / Accepted: 31 August 2015 / Published: 9 September 2015

\begin{abstract}
Conditions for load-independent output voltage or current in two-coil wireless power transfer (WPT) systems have been studied. However, analysis of load-independent output current in three-coil WPT system is still lacking in previous studies. This paper investigates the output current characteristics of a three-coil WPT system against load variations, and determines the operating frequency to achieve a constant output current. First, a three-coil WPT system is modeled by circuit theory, and the analytical expression of the root-mean-square of the output current is derived. By substituting the coupling coefficients, the quality factor, and the resonant frequency of each coil, we propose a method of calculating the frequency for load-independent output current in a three-coil WPT system, which indicates that there are two frequencies that can achieve load-independent output current. Experiments are conducted to validate these analytical results.
\end{abstract}

Keywords: three-coil; wireless power transfer (WPT); frequency; load-independent output current

\section{Introduction}

Nowadays, with its considerable potential for practical applications, wireless power transfer (WPT) technology is gaining much attention from researchers. WPT system can deliver electrical energy from 
a power source to a load via varying magnetic fields, and the transmission power level ranges from a few milliwatts to several hundred kilowatts.

There are many benefits that make this technique particularly attractive, such as the electrical and mechanical isolation, the decrease in maintenance costs, and the potential for use in harsh environments. Therefore, WPT can be used for wireless battery charging for implantable biomedical devices [1-4] and portable electronics [5-7]. Moreover, even electric vehicles (or roadway-powered electric vehicles) can be wirelessly powered [8-11].

In terms of practical applications, most of the circuits and systems require constant source supply for stable operation; therefore, a relatively constant output in WPT system is desired regardless of load variations.

In two-coil WPT systems, there have been many efforts [1,2,12-17] to regulate the output voltage under varying couplings and loads. [12-15] exhibited relatively constant output voltage under coupling variations. However, the variation of output voltage is relatively high, and the distance range for constant output is limited. In addition, the effect of load variations on output voltage is not discussed. In [1], using the transformer model, the voltage transfer function was obtained; an operating frequency corresponding to a particular coupling coefficient could be found, for which the voltage transfer function was irrelevant to load. In [2], the output voltage was insensitive to loading variations if the operating frequency was adjusted according to coupling variations, and a parallel-resonant class-D oscillator transmitter was developed to track this optimal operating frequency. In [16], a design and optimization method was proposed to achieve a better overall efficiency and good output voltage controllability in a WPT system. For both the secondary series- and parallel-compensations in two-coil WPT, by calculating the partial derivative of the voltage gain with respect to the load, [17] analyzed the frequencies that could provide a constant voltage gain despite load variations.

However, in some charging applications, a load-independent output current is more desirable. For example, a constant output current is preferred for driving an LED to have stable luminance, or multistep constant current of a charging profile to charge a battery pack for electric vehicles. An extra current regulator stage, which results in extra power loss, is needed if the WPT system outputs as a voltage source. In [18], operating frequencies for maximal efficiency and load-independent output current in two-coil WPT are explored. Moreover, the dual circuits of the secondary series- and parallel-compensated WPT have been studied. It is found that the trend of the output current of a series-compensated (parallel-compensated) WPT system studied in [18] replicates the trend of the output voltage of a parallel-compensated (series-compensated) WPT system in [17].

With increasing transmission distance in two-coil WPT, the performance of the two-coil WPT deteriorates. Adding a repeating coil, which receives the magnetic field from the transmitter and then relays the magnetic field to the receiver, enhances inductive coupling at longer distances. However, a detailed analysis of load-independent output current in such a three-coil WPT system is still lacking in previous studies.

In this study, a three-coil WPT system is investigated. To determine the operating frequency for the particular coupling coefficient - in this case, the output current remains constant despite changes in the load - we first substitute the coupling coefficients, the quality factor, and the resonant frequency of each coil into the expression of the root-mean-square (RMS) of the output current, and then the frequencies 
that can provide load-independent output current in a three-coil WPT are found for the first time. To verify the results of this analysis, theoretical calculations and experiments are conducted.

\section{Modeling}

Figure 1 presents a photograph of a three-coil WPT system. The AC voltage source supplies power for the transmitting coil (TX). Through the inductive coupling between the coils, current can be induced in the receiving coil $(\mathrm{RX})$, providing power for the load $[19,20]$.

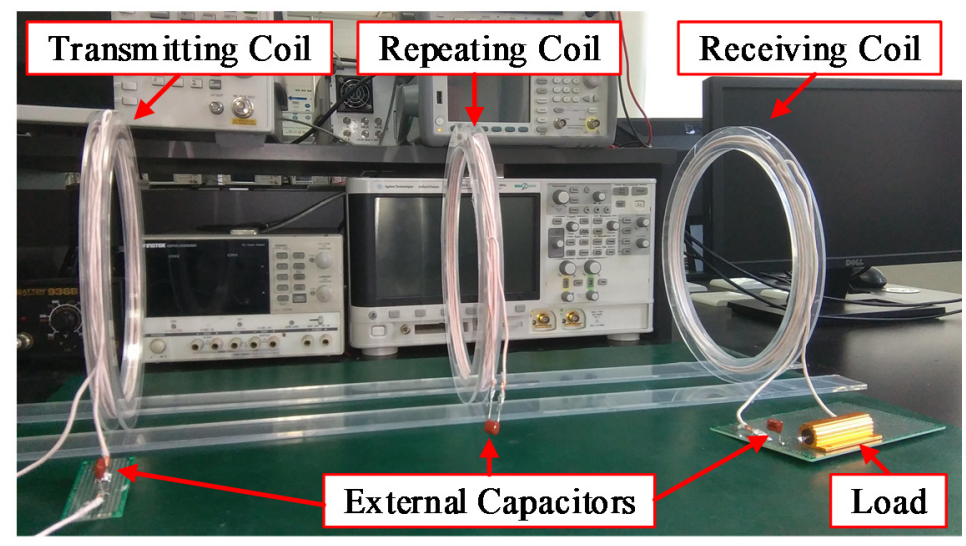

Figure 1. Photograph of a three-coil WPT system.

As shown in Figure 2, the WPT system can be modeled by circuit theory [21,22]. A constant AC voltage source $V_{\text {in }}$ is used for the power supply, and $R_{L}$ is the loading resistor. The TX, the repeating coil, and the RX are represented as a resistor-inductor-capacitor (RLC) circuit, and the inductive coupling between the coils is described by the mutual inductance. The parameters of the TX are denoted with subscript 1 , the repeating coil with subscript 2 , and the RX with subscript $3 . L_{i}$ is the inductance of coil $i(i=1,2,3)$, whose equivalent resistance is $R_{i}$, and external capacitor $C_{i}$ is added to form a series-tuned topology. $M_{12}$ and $M_{23}$ are the mutual inductances between each two adjacent coils, and $M_{13}$ is the mutual inductance between the TX and the RX.

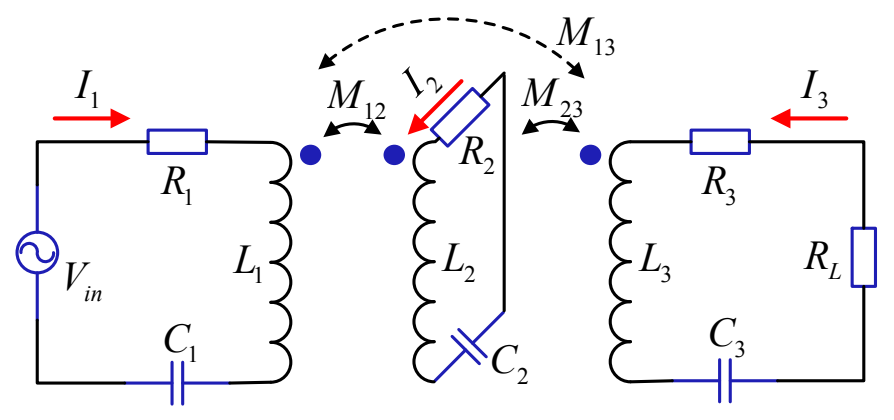

Figure 2. Equivalent circuit of the three-coil WPT system. 


\section{Frequency for Load-Independent Output Current in Three-Coil WPT}

The Kirchhoff's voltage law (KVL) equations of the equivalent circuit in Figure 2 are:

$$
\left[\begin{array}{ccc}
R_{1}+j \omega L_{1}+\frac{1}{j \omega C_{1}} & j \omega M_{12} & j \omega M_{13} \\
j \omega M_{12} & R_{2}+j \omega L_{2}+\frac{1}{j \omega C_{2}} & j \omega M_{23} \\
j \omega M_{13} & j \omega M_{23} & R_{3}+j \omega L_{3}+\frac{1}{j \omega C_{3}}+R_{L}
\end{array}\right]\left[\begin{array}{c}
\mathbf{I}_{1} \\
\mathbf{I}_{2} \\
\mathbf{I}_{3}
\end{array}\right]=\left[\begin{array}{c}
\mathbf{V}_{\text {in }} \\
0 \\
0
\end{array}\right]
$$

where $\mathbf{I}_{\mathbf{i}}$ is the current in the coil $i(i=1,2,3), \mathbf{V}_{\text {in }}$ is the voltage source, and $\omega$ is the operating frequency.

In this study, bold letters are used to represent the phasors, and italic letters denote real numbers or RMS values of the phasors. For example, $\mathbf{I}_{\mathbf{1}}$ is a current phasor, and $I_{1}$ is the RMS value of $\mathbf{I}_{1}$. For the given three-coil WPT system, the equivalent resistances of the coils are small enough to be omitted. For simplicity, the cross-coupling $M_{13}$ is neglected in comparison to the coupling between adjacent coils $M_{12}$ and $M_{23}$.

The expression of the output current $\mathbf{I}_{3}$ can be obtained by solving Equation (1). To facilitate the analysis, six factors are introduced: the coupling coefficients $k_{12}$ and $k_{23}$, the quality factor $Q$, the resonant frequency $\omega_{i}(i=1,2,3)$ of the coil $i$. These factors are, respectively, defined as:

$$
k_{12}=M_{12} / \sqrt{L_{1} L_{2}}, \quad k_{23}=M_{23} / \sqrt{L_{2} L_{3}}, \quad Q=\omega_{3} L / R_{L}, \quad \omega_{i}=1 / \sqrt{L_{i} C_{i}}(i=1,2,3)
$$

By substituting Equation (2) into the expression of $\mathbf{I}_{3}$, the RMS of $\mathbf{I}_{3}$ can be derived by:

$$
I_{3}=\frac{\sqrt{C_{1} C_{3}} k_{12} k_{23} \omega^{3} V_{\mu n}}{\omega_{1} \omega_{2}^{2} \omega_{3} \sqrt{\left(\frac{\omega^{2}}{\omega_{1}^{2}}+\frac{\omega^{2}}{\omega_{2}^{2}}+\frac{\omega^{2}}{\omega_{3}^{2}}-\frac{\left(1-k_{12}^{2}\right) \omega^{4}}{\omega_{1}^{2} \omega_{2}^{2}}-\frac{\left(1-k_{23}^{2}\right) \omega^{4}}{\omega_{1}^{2} \omega_{3}^{2}}-\frac{\omega^{4}}{\omega_{2}^{2} \omega_{3}^{2}}+\frac{\left(1-k_{12}^{2}-k_{23}^{3}\right) \omega^{6}}{\omega_{1}^{2} \omega_{2}^{2} \omega_{3}^{2}}-1\right)^{2}+\frac{\omega^{2}}{Q^{2} \omega_{3}^{2}}\left(\frac{\left(1-k_{12}^{2}\right) \omega^{4}}{\omega_{1}^{2} \omega_{2}^{2}}-\frac{\omega^{2}}{\omega_{1}^{2}}-\frac{\omega^{2}}{\omega_{2}^{2}}+1\right)^{2}}}
$$

From Equation (3), it can be readily observed that if:

$$
\frac{\left(1-k_{12}^{2}\right) \omega^{4}}{\omega_{1}^{2} \omega_{2}^{2}}-\frac{\omega^{2}}{\omega_{1}^{2}}-\frac{\omega^{2}}{\omega_{2}^{2}}+1=0
$$

then the output current in Equation (3) can be rewritten as:

$$
I_{3}=\frac{\sqrt{C_{1} C_{3}} k_{12} k_{23} \omega^{5} V_{i n}}{\omega_{1} \omega_{2}{ }^{2} \omega_{3}\left|\frac{\omega^{2}}{\omega_{1}{ }^{2}}+\frac{\omega^{2}}{\omega_{2}{ }^{2}}+\frac{\omega^{2}}{\omega_{3}{ }^{2}}-\frac{\left(1-k_{12}{ }^{2}\right) \omega^{4}}{\omega_{1}{ }^{2} \omega_{2}{ }^{2}}-\frac{\left(1-k_{23}{ }^{3}\right) \omega^{4}}{\omega_{1}{ }^{2} \omega_{3}{ }^{2}}-\frac{\omega^{4}}{\omega_{2}{ }^{2} \omega_{3}{ }^{2}}+\frac{\left(1-k_{12}{ }^{2}-k_{23}{ }^{3}\right) \omega^{6}}{\omega_{1}{ }^{2} \omega_{2}{ }^{2} \omega_{3}{ }^{2}}-1\right|}
$$

which is insensitive to $Q$. Due to the fact that $Q$ is inversely proportional to $R_{L}, I_{3}$ in Equation (5) is insensitive to $R_{L}$. This load-independent output current is a very desirable feature in three-coil WPT system. This guarantees constant output current when the load changes without using control circuit.

By solving Equation (4), we can obtain the frequencies for load-independent output current in three-coil WPT. Because $1-k_{12}{ }^{2}>0$ holds, and the discriminant (i.e., $\left.\Delta=\left(\omega_{1}{ }^{2}-\omega_{2}{ }^{2}\right)^{2}+4 k_{12}{ }^{2} \omega_{1}{ }^{2} \omega_{2}{ }^{2}\right)$ in 
Equation (4) is positive, according to Vieta's formulas, the bi-quadratic Equation (4) has two positive roots:

$$
\omega=\left[\frac{\left(\omega_{1}^{2}+\omega_{2}^{2}\right) \pm \sqrt{\left(\omega_{1}^{2}-\omega_{2}^{2}\right)^{2}+4 k_{12}{ }^{2} \omega_{1}^{2} \omega_{2}^{2}}}{2\left(1-k_{12}{ }^{2}\right)}\right]^{0.5}
$$

where the smaller one is $\omega_{-}$and the larger one is $\omega_{+}$.

Specifically, when the resonant frequencies of the coils are identical, that is, $\omega_{1}=\omega_{2}=\omega_{3}=\omega_{0}$, substituting $\omega_{1}=\omega_{2}=\omega_{3}=\omega_{0}$ into Equation (6), the frequencies for load-independent output current in a three-coil WPT in Equation (6) can be reduced as:

$$
\omega=\frac{\omega_{0}}{\sqrt{1 \pm k_{12}}}
$$

The expressions of the RMS of the currents in the TX, repeating coil, and RX (i.e., $I_{1}, I_{2}$, and $I_{3}$ ) can be obtained by solving Equation (1). Thus, the transfer efficiency $\eta$ in a three-coil WPT system is defined as the ratio of the power dissipation in the load and the total power supplied by the source [23-25]:

$$
\eta=\frac{I_{3}^{2} R_{L}}{I_{1}^{2} R_{1}+I_{2}^{2} R_{2}+I_{3}^{2}\left(R_{3}+R_{L}\right)}
$$

The output power $P_{\text {out }}$ in three-coil WPT system is calculated by:

$$
P_{\text {out }}=I_{3}^{2} R_{L}
$$

Figures 3 and 4 show how the calculated output current varies with the operating frequency under three loading conditions in a three-coil WPT system with the parameters listed in Table 1 . The coupling coefficients shown in Figure 3 are $k_{12}=0.1$ and $k_{23}=0.2$, and that in Figure 4 are $k_{12}=0.5$ and $k_{23}=0.4$.

Table 1. Parameters of the experimental setup.

\begin{tabular}{ccc}
\hline Symbol & Note & Value \\
\hline$V_{\text {in }}$ & RMS source voltage & $30 \mathrm{~V}$ \\
$f_{1}, f_{2}, f_{3}$ & Resonant frequency of coil 1,2,3 & $105 \mathrm{kHz}, 100 \mathrm{kHz}, 95 \mathrm{kHz}$ \\
$L_{1}, L_{2}, L_{3}$ & Inductance of coil 1, 2,3 & $58.91 \mathrm{uH}, 37.25 \mathrm{uH}, 50.12 \mathrm{uH}$ \\
$C_{1}, C_{2}, C_{3}$ & Capacitance of coil 1,2,3 & $39 \mathrm{nF}, 68 \mathrm{nF}, 56 \mathrm{nF}$ \\
$R_{1}, R_{2}, R_{3}$ & Resistance of coil 1,2,3 & $0.50 \Omega, 0.39 \Omega, 0.46 \Omega$ \\
$R_{L}$ & Load resistance & $12 \Omega, 18 \Omega, 32 \Omega$ \\
\hline
\end{tabular}




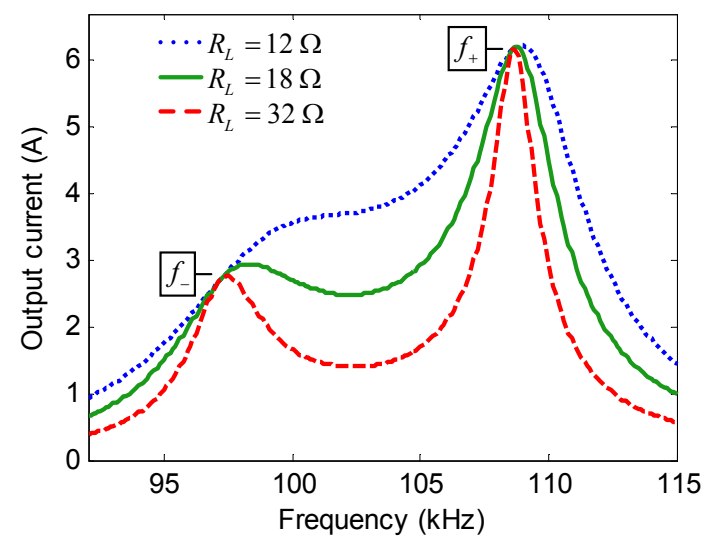

Figure 3. Calculated output current versus frequency in a three-coil WPT when $k_{12}=0.1$ and $k_{23}=0.2$.

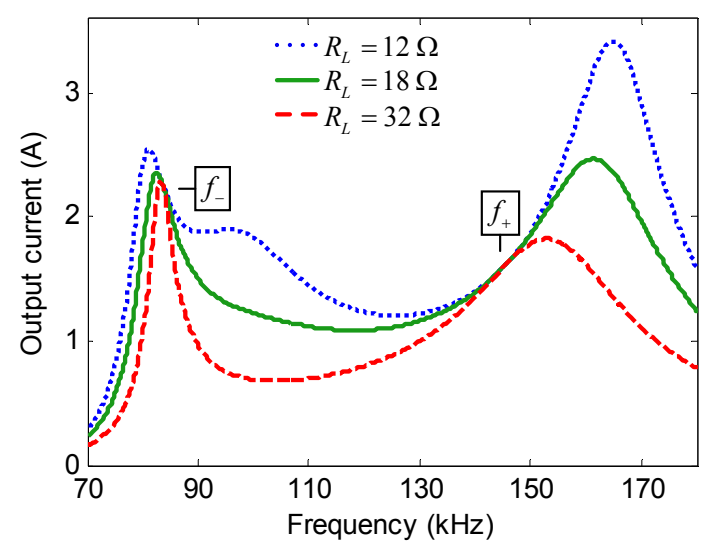

Figure 4. Calculated output current versus frequency in a three-coil WPT when $k_{12}=0.5$ and $k_{23}=0.4$.

As presented in Figures 3 and 4, for the given coupling coefficients $k_{12}$ and $k_{23}$, there are two operating frequencies at which $I_{3}$ is irrelevant to $R_{L}$. In Figure $3, f_{-}=\omega_{-} / 2 \pi=97.15 \mathrm{kHz}$ and $f_{+}=\omega_{+} / 2 \pi=108.62 \mathrm{kHz}$; in Figure $4, f_{-}=\omega_{-} / 2 \pi=83.57 \mathrm{kHz}$ and $f_{+}=\omega_{+} / 2 \pi=145.09 \mathrm{kHz}$.

\section{Experiments}

To verify the above analysis for a three-coil WPT, an experimental setup is implemented, and a set of experiments are performed. Three polypropylene capacitor with the capacitance value $39 \mathrm{nF}, 56 \mathrm{nF}$, and $68 \mathrm{nF}$ are used as the external capacitors. The three-coil WPT system is composed of three circular coils with a radius of $100 \mathrm{~mm}$. These coils are fabricated with Litz wire, and the radius of the Litz wire used in the TX, the repeating coil, and the RX are $1.3 \mathrm{~mm}, 1.3 \mathrm{~mm}$, and $1.0 \mathrm{~mm}$, respectively. The turn number of the Litz wire used in the TX, the repeating coil, and the RX are 10, 8, and 9, respectively. The inductance of each coil can be measured by an LCR meter. Figure 1 displays the photograph of the experimental setup, whose parameters are listed in Table 1. Standard sinusoidal signals generated by a signal generator are amplified by a power amplifier and fed to the transmitting coil of the three-coil WPT system. 
For two coaxial circular coils, the mutual inductance can be calculated using Equation $(10)[25,26]$

$$
M=\mu_{0} N_{1} N_{2} \sqrt{r_{1} r_{2}}\left[\left(\frac{2}{\gamma}-\gamma\right) K(\gamma)-\frac{2}{\gamma} E(\gamma)\right]
$$

where $\gamma=\sqrt{4 r_{1} r_{2} /\left(\left(r_{1}+r_{2}\right)^{2}+d^{2}\right)}, K(\gamma)$ and $E(\gamma)$ are complete elliptic integrals of the first and second kind, respectively, and the values of $K(\gamma)$ and $E(\gamma)$ can be obtained by Sidhu's method, as elucidated in [27]; $\mu_{0}$ is the permeability of vacuum, $N_{1}$ and $N_{2}$ are the number of turns of the two coils, $r_{1}$ and $r_{2}$ are the radius of coil-1 and coil-2, respectively, and $d$ is the distance between the two coils.

The inductances of the coils are measured by an LCR meter, and the mutual inductance between the coils is calculated by Equation (10); thus, the coupling coefficients can be calculated by Equation (2).

Figures 5 and 6 show the measured output current versus the operating frequency under three loading conditions in a three-coil WPT.

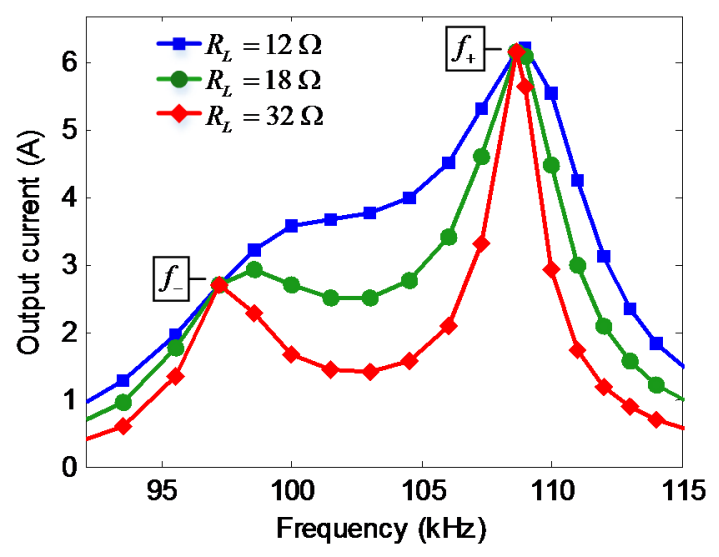

Figure 5. Measured output current versus frequency in a three-coil WPT when $k_{12}=0.1$ and $k_{23}=0.2$.

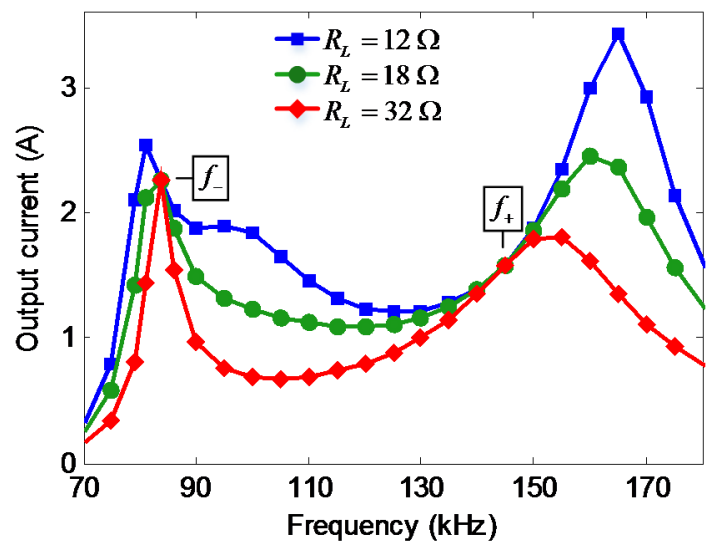

Figure 6. Measured output current versus frequency in a three-coil WPT when $k_{12}=0.5$ and $k_{23}=0.4$.

As shown in Figure 5, when $k_{12}=0.1$ and $k_{23}=0.2$, there are two operating frequencies, i.e., $f_{-}=97.3 \mathrm{kHz}$ and $f_{+}=108.5 \mathrm{kHz}$, at which $I_{3}$ is insensitive to $R_{L}$; these findings are consistent with the calculated results in Figure 3. 
In Figure 6, when $k_{12}=0.5$ and $k_{23}=0.4$, the frequencies for load-independent output current are $f_{-}=83.7 \mathrm{kHz}$ and $f_{+}=145.0 \mathrm{kHz}$, which agree with the calculated results in Figure 4.

Upon comparing the trend of the output current in Figures 5 and 6 with that in Figures 3 and 4, respectively, the measured results confirm the calculated results.

Furthermore, Equation (6) demonstrates that the frequency for load-independent output current is a function of the coupling coefficient $k_{12}$ and irrelevant to $k_{23}$, that is, when $k_{12}$ is given, the frequencies for load-independent output current in a three-coil WPT are determined.

Figure 7 presents the calculated and measured frequencies for load-independent output current versus the coupling coefficient $k_{12}$ in a three-coil WPT. The dotted line and dashed line are the calculated values of $f_{-}$and $f_{+}$, respectively. The points are measured data: the circles and diamonds denote $f_{-}$and $f_{+}$, respectively. The calculated and measured results are in good agreement, which validates the conclusion reached in Section 3.

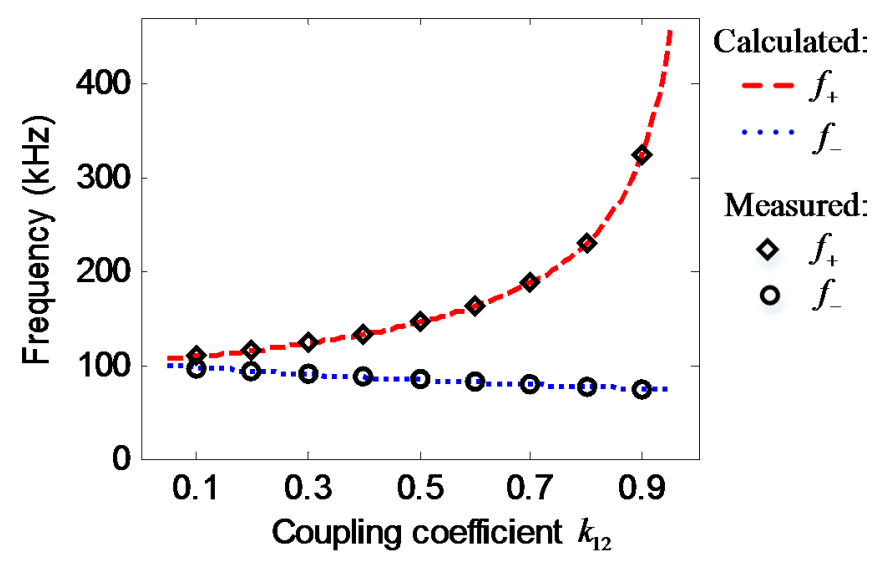

Figure 7. Calculated and measured frequencies for load-independent output current versus coupling coefficient $k_{12}$ in a three-coil WPT.

When $k_{12}=0.1$ and $k_{23}=0.2$, the measured results of output power and transfer efficiency versus frequency in a three-coil WPT system are displayed in Figure 8a,b, respectively. When $k_{12}=0.5$ and $k_{23}=0.4$, Figure $9 \mathrm{a}, \mathrm{b}$ reveal how the measured output power and transfer efficiency vary with frequency in a three-coil WPT system, respectively.

As shown in Figure 8a, in the case of the small coupling coefficients (i.e., at long distances), when the load resistance is relatively small, there is only peak value of the output power; when the load resistance grows, that is, the quality factor decreases, two peaks of the output power arise because of the frequency splitting [23]. Figure 9a indicates that when the coupling coefficients are relatively large (i.e., at close distances), there are two peak values of the output power, which is called the frequency splitting phenomenon [23]. 


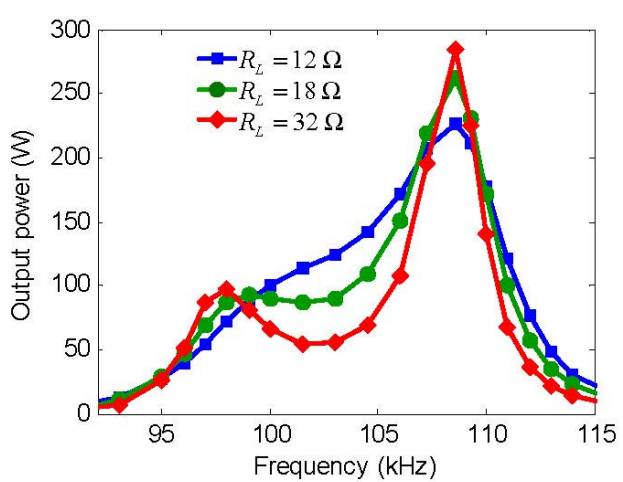

(a)

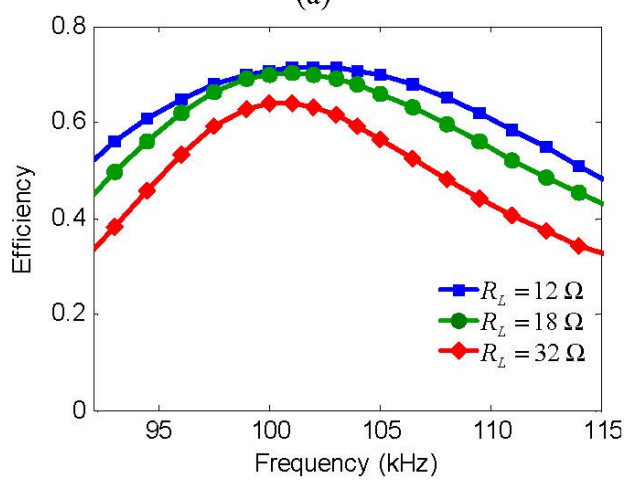

(b)

Figure 8. Measured results versus frequency in a three-coil WPT when $k_{12}=0.1$ and $k_{23}=0.2:$ (a) output power; and (b) efficiency.

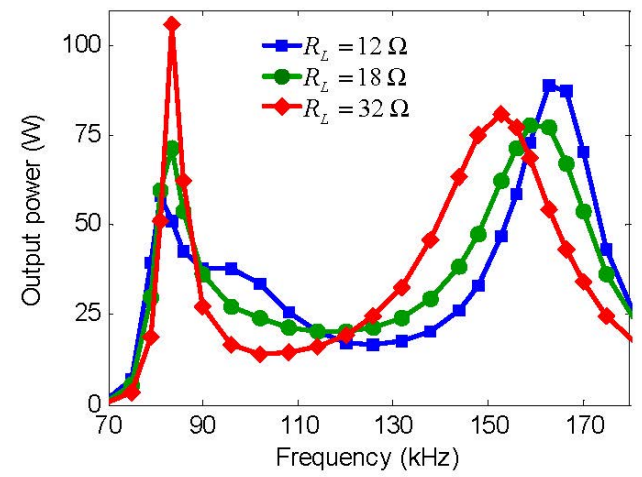

(a)

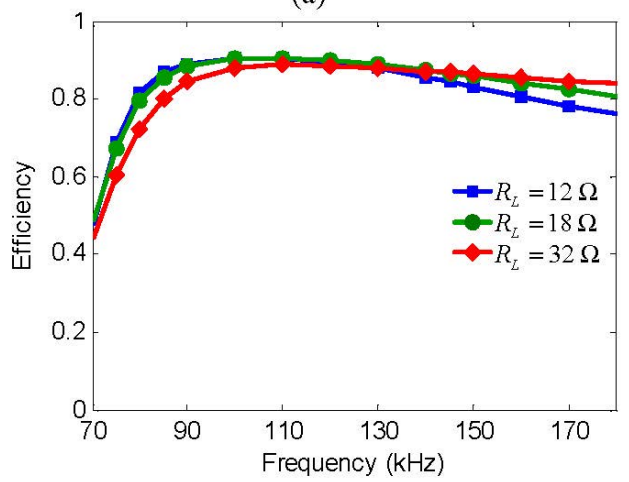

(b)

Figure 9. Measured results versus frequency in a three-coil WPT when $k_{12}=0.5$ and $k_{23}=0.4:$ (a) output power; and (b) efficiency. 
When the load resistance varies, the transfer efficiency in the three-coil WPT system varies accordingly, that is, there exists an optimal load to obtain the maximal transfer efficiency. Comparing Figure $8 \mathrm{~b}$ with Figure $9 \mathrm{~b}$, we observe that increasing the coupling coefficients (i.e., at close distances) results in higher transfer efficiency.

\section{Conclusions}

Based on circuit theory, load-independent output current in a three-coil WPT system is thoroughly studied. By introducing six factors, namely, the coupling coefficients ( $k_{12}$ and $k_{23}$ ), the quality factor $(Q)$, and the resonant frequency of each coil $\left(\omega_{1}, \omega_{2}\right.$, and $\left.\omega_{3}\right)$, and substituting these factors into the expression of the RMS of the output current, we propose an intuitive method of calculating the frequency for load-independent output current, which indicates that there are two frequencies that can achieve load-independent output current in three-coil WPT. An experimental setup is implemented, and the measured results show excellent agreement with the theoretical analysis. Furthermore, the measured results of output power and transfer efficiency in a three-coil WPT system are also presented.

In comparison with previous study [18], this study can assist us in achieving a load-independent output current at longer distances. The proposed method and conclusion provide guidelines for selecting the optimal operating frequency in three-coil WPT system to obtain a constant output current under varying load conditions, which is a significant progress toward practical applications for WPT technology.

\section{Acknowledgments}

This work was supported by the National Natural Science Foundation of China (51277120).

\section{Author Contributions}

Longzhao Sun proposed the main idea, performed the experiments, and wrote the manuscript. Houjun Tang contributed to the discussion of this study. Yingyi Zhang double-checked the results and the whole manuscript. All authors have approved the final version of this manuscript.

\section{Conflicts of Interest}

The authors declare no conflict of interest.

\section{References}

1. Chen, Q.; Wong, S.C.; Tse, C.K.; Ruan, X. Analysis, design, and control of a transcutaneous power regulator for artificial hearts. IEEE Trans. Biomed. Circuits Syst. 2009, 3, $23-31$.

2. Ahn, D.; Hong, S. Wireless power transmission with self-regulated output voltage for biomedical implant. IEEE Trans. Ind. Electron. 2014, 61, 2225-2235.

3. Nair, V.V.; Choi, J.R. An integrated chip high-voltage power receiver for wireless biomedical implants. Energies 2015, 8, 5467-5487.

4. Ho, J.S.; Kim, S.; Poon, A.S.Y. Midfield wireless powering for implantable systems. IEEE Proc. 2013, 101, 1369-1378. 
5. Zhong, W.X.; Liu, X.; Hui, S.R. A novel single-layer winding array and receiver coil structure for contactless battery charging systems with free-positioning and localized charging features. IEEE Trans. Ind. Electron. 2011, 58, 4136-4144.

6. Hui, S.Y. Planar wireless charging technology for portable electronic products and Qi. IEEE Proc. 2013, 101, 1290-1301.

7. Cheng, S.J.; Chiu, H.J.; Lo, Y.K.; Kuo, S.W.; Jen, K.K.; Fu, K.S.; You, G.H.; Chen, K.F.; Kao, C.M. Design and implementation of a contact-less power charger for robot applications. Int. J. Circuit Theory Appl. 2014, 42, 584-604.

8. Zhang, Z.; Chau, K.T.; Liu, C.; Qiu, C.; Ching, T.W. A positioning-tolerant wireless charging system for roadway-powered electric vehicles. J. Appl. Phys. 2015, 117, 17B520, doi:10.1063/1.4916187.

9. Zhang, Z.; Chau, K.T.; Liu, C.; Qiu, C.; Lin, F. An efficient wireless power transfer system with security considerations for electric vehicle applications. J. Appl. Phys. 2014, 115, 17A328, doi:10.1063/1.4866238.

10. Covic, G.A.; Boys, J.T. Modern trends in inductive power transfer for transportation applications. IEEE J. Emerg. Sel. Top. Power Electron. 2013, 1, 28-41.

11. Li, W.; Mi, C.C.; Li, S.; Deng, J.; Kan, T.; Zhao, H. Integrated LCC compensation topology for wireless charger in electric and plug-in electric vehicles. IEEE Trans. Ind. Electron. 2015, 62, 4215-4225.

12. Zierhofer, C.M.; Hochmair, E.S. High-efficiency coupling insensitive transcutaneous power and data transmission via an inductive link. IEEE Trans. Biomed. Eng. 1990, 37, 716-722.

13. Baker, M.W.; Sarpeshkar, R. Feedback analysis and design of RF power links for low-power bionic systems. IEEE Trans. Biomed. Circuits Syst. 2007, 1, 28-38.

14. Villa, J.; Sallan, J.; Osorio, J.; Llombart, A. High-misalignment tolerant compensation topology for ICPT systems. IEEE Trans. Ind. Electron. 2012, 59, 945-951.

15. Zierhofer, C.M.; Hochmair, E.S. The class-E concept for efficient wide-band coupling-insensitive transdermal power and data transfer. IEEE Proc. Annu. Int. Conf. 1992, 2, 382-383.

16. Zhang, W.; Wong, S.C.; Tse, C.K.; Chen, Q. Design for efficiency optimization and voltage controllability of series-series compensated inductive power transfer systems. IEEE Trans. Power Electron. 2014, 29, 191-200.

17. Zhang, W.; Wong, S.C.; Tse, C.K.; Chen, Q. Analysis and comparison of secondary series- and parallel-compensated inductive power transfer systems operating for optimal efficiency and load-independent voltage-transfer ratio. IEEE Trans. Power Electron. 2014, 29, 2979-2990.

18. Zhang, W.; Wong, S.C.; Tse, C.K.; Chen, Q. Load-independent duality of current and voltage outputs of a series- or parallel-compensated inductive power transfer converter with optimized efficiency. IEEE J. Emerg. Sel. Top. Power Electron. 2015, 3, 137-146.

19. Pinuela, M.; Yates, D.C.; Lucyszyn, S.; Mitcheson, P.D. Maximizing DC-to-load efficiency for inductive power transfer. IEEE Trans. Power Electron. 2013, 28, 2437-2447.

20. Puccetti, G.; Reggiani, U.; Sandrolini, L. Experimental analysis of wireless power transmission with spiral resonators. Energies 2013, 6, 5887-5896.

21. Wei, X.; Wang, Z.; Dai, H. A critical review of wireless power transfer via strongly coupled magnetic resonances. Energies 2014, 7, 4316-4341. 
22. Kiani, M.; Ghovanloo, M. The circuit theory behind coupled-mode magnetic resonance-based wireless power transmission. IEEE Trans. Circuits Syst. Part I Regul. Pap. 2012, 59, 2065-2074.

23. Ahn, D.; Hong, S. A study on magnetic field repeater in wireless power transfer. IEEE Trans. Ind. Electron. 2013, 60, 360-371.

24. Zhang, Y.; Zhao, Z.; Lu, T. Quantitative analysis of system efficiency and output power of four-coil resonant wireless power transfer. IEEE J. Emerg. Sel. Top. Power Electron. 2015, 3, 184-190.

25. Lee, S.H.; Lorenz, R.D. Development and validation of model for $95 \%$ efficiency $220 \mathrm{~W}$ wireless power transfer over a 30-cm air gap. IEEE Trans. Ind. Appl. 2011, 47, 2495-2504.

26. Zhang, X.; Ho, S.L.; Fu, W.N. Quantitative design and analysis of relay resonators in wireless power transfer system. IEEE Trans. Magn. 2012, 48, 4026-4029.

27. Miller, H.C. Inductance formula for a single-layer coil. IEEE Proc. 1987, 75, 256-257.

(C) 2015 by the authors; licensee MDPI, Basel, Switzerland. This article is an open access article distributed under the terms and conditions of the Creative Commons Attribution license (http://creativecommons.org/licenses/by/4.0/). 